\title{
HOLOMORPHIC APPROXIMATION ON COMPACT PSEUDOCONVEX COMPLEX MANIFOLDS
}

\author{
Hong Rae $\mathrm{CHO}^{\dagger}$ And SAnghyun ChO
}

\begin{abstract}
Let $\bar{M}$ be a smoothly bounded compact pseudoconvex complex manifold of finite type in the sense of D'Angelo such that the complex structure of $M$ extends smoothly up to $b M$. Let $m$ be an arbitrary nonnegative integer. Let $f$ be a function in $H(M) \cap H_{m}(M)$, where $H_{m}(M)$ is the Sobolev space of order $m$. Then $f$ can be approximated by holomorphic functions on $\bar{M}$ in the Sobolev space $H_{m}(M)$. Also, we get a holomorphic approximation theorem near a boundary point of finite type.
\end{abstract}

\section{Introduction and statement of the results.}

In [14], Mergelyan proved that if $K \subset \mathbb{C}$ is compact and $\mathbb{C}-K$ has just one connected component, then every $f \in C(K)$ which is holomorphic in the interior of $K$ can be approximated uniformly on $K$ by functions holomorphic in some neighborhood of $K$ (and hence by polynomials). One cannot obtain the Mergelyan approximation property in several complex variables only with the simple topological hypothesis. Surprisingly, Diederich and Fornaess [8] constructed a smoothly bounded pseudoconvex domain $D$ in $\mathbb{C}^{2}$ and a function $f \in A^{\infty}(D)=H(D) \cap C^{\infty}(\bar{D})$ which can not be uniformly approximated by functions in $H(\bar{D})$. However, there is a classical result that proves the Mergelyan approximation property:

Theorem (Kerzman [12], Henkin [11], Lieb [13]). Let $D \Subset \mathbb{C}^{n}$ be a smoothly bounded strongly pseudoconvex domain. Then any function continuous on $\bar{D}$ and holomorphic on $D$ can be approximated uniformly on $\bar{D}$ by functions holomorphic on $\bar{D}$.

In the weakly pseudoconvex case, Fornaess and Nagel [9] got the same result when $D \Subset \mathbb{C}^{2}$ is a pseudoconvex domain with real analytic boundary. Other generalizations can be found in $[1,15]$. The purpose of this paper is to get the Mergelyan approximation property on compact pseudoconvex complex manifolds of finite type in the sense of D'Angelo [7]. However, instead of using the uniform convergence topology, we use the topology given in Sobolev spaces.

Let $\bar{M}$ be a smoothly bounded compact manifold and $f \in L^{2}(M)$. Let $\zeta_{j}, j=$ $1,2, \ldots, N$, be a partition of unity subordinated to a covering of $\bar{M}$. We define

$$
\|f\|_{m, M}^{2}=\sum_{j=1}^{N} \sum_{|\alpha| \leq m}\left\|D^{\alpha}\left(\zeta_{j} f\right)\right\|^{2}
$$

2000 Mathematics Subject Classification. Firstly 32E30, Secondly 32F20.

Key words and phrases. holomorphic approximation, finite type, $\bar{\partial}$-Neumann problem.

$\ddagger$ Partially supported by Korea Research Foundation (1998), and GARC-KOSEF (1998) 
where $D^{\alpha}$ refers to $\partial^{|\alpha|} / \partial x_{1}^{\alpha_{1}} \cdots \partial x_{2 n}^{\alpha_{2 n}}$ for the coordinate neighborhood of supp $\zeta_{j}$. Then we let the $m$-th order Sobolev space, $H_{m}(M)$, be the space of all $f \in L^{2}(M)$ such that $\|f\|_{m, M}<\infty$.

In [4], Catlin proved the existence of local holomorphic coordinate functions up to the boundary in case $\bar{M}$ is a pseudoconvex complex manifold. In the sense of D'Angelo, finite type depends on the finite order of Taylor polynomial of the defining function for $\bar{M}$. Therefore, it is reasonable to define a finite type for the manifolds in conjunction with holomorphic coordinates.

Definition 1.1. Let $\bar{M}$ be a compact pseudoconvex complex manifold and $z_{0} \in$ $b M$. Then we have a neighborhood $U$ of $z_{0}$ and holomorphic coordinate functions $f=\left(f_{1}, \ldots, f_{n}\right)$ defined on $U$. In this case, we define; Type of $z_{0}=$ Type of $f\left(z_{0}\right)$ on $f(b M)$ in $\mathbb{C}^{n}$.

Since the type condition is an invariant property, it is clear that the above definition of type does not depend on coordinate functions. For compact pseudoconvex complex manifold $\bar{M}$ of finite type ( $\operatorname{dim}_{\mathbb{C}} M=n \geq 2$ ), the second author [6] showed that $\bar{M}$ can be holomorphically embedded into a larger complex manifold $\Omega$ of the same complex dimension. In virtue of this result, we can now state our main theorems as follows:

Theorem 1.1. Let $\bar{M}$ be a compact pseudoconvex complex manifold with smooth boundary. Assume also that $b M$ is finite type and $\operatorname{dim}_{\mathbb{C}} M \geq 2$. Let $f$ be a function in $H(M) \cap H_{m}(M)$, where $m$ is a nonnegative integer. Then there exists a sequence of functions $f_{n}$ in $H(\bar{M})$ such that

$$
\lim _{n \rightarrow \infty}\left\|f_{n}-f\right\|_{m, M}=0
$$

Among the many uses of the holomorphic approximation theorem, we mention the construction of peak functions [2] and the stability of the Bergman kernel function [10]. A result on the interior stability of the Bergman kernel function is given in Section 3.

Also, we get a holomorphic approximation theorem near a boundary point of finite type.

Theorem 1.2. Let $\bar{M}$ and $r$ be as in Theorem 1.1 without the assumption of finite type. Suppose there is a $C^{\infty}$ plurisubharmonic function $\varphi$ on $\bar{M}$ which is strongly plurisubharmonic on a neighborhood of $b M$. Let $z_{0}$ be a point of finite type in the boundary of $M$. Let $f$ be a function in $H(M) \cap H_{m}(M)$, where $m$ is a nonnegative integer. Then there exist a neighborhood $V$ of $z_{0}$ and a sequence of functions $f_{n}$ in $A^{\infty}(M)$ which extend holomorphically on $V \cap b \Omega$ such that

$$
\lim _{n \rightarrow \infty}\left\|f_{n}-f\right\|_{m, M}=0
$$

Now let $D$ be a smoothly bounded pseudoconvex domain in $\mathbb{C}^{n}$. Then $\varphi(z)=$ $|z|^{2}$ is a $C^{\infty}$ strongly plurisubharmonic function on $\bar{D}$. Therefore, we obtain the following immediate consequence of Theorem 1.2.

Corollary 1.3. Let $z_{0}$ be a point of finite type in the boundary of D. Let $f$ be a function in $H(D) \cap H_{m}(D)$, where $m$ is a nonnegative integer. Then there exist 
a neighborhood $V$ of $z_{0}$ and a sequence of functions $f_{n}$ in $A^{\infty}(D)$ which extend holomorphically on $V \cap b \Omega$ such that

$$
\lim _{n \rightarrow \infty}\left\|f_{n}-f\right\|_{m, M}=0 .
$$

\section{A stability theorem for the $\bar{\partial}$-Neumann problem.}

Let $\bar{M}$ be as in Theorem 1.1 and let $M^{\prime} \supset \bar{M}$ be a $C^{\infty}$ manifold. Assume that there exist a neighborhood $V$ of $b M$ and a real-valued function $r \in C^{\infty}(V)$ such that $\nabla r \neq 0$ on $b M$ and $r(z)=0$ if and only if $z \in b M$. The sign of $r$ is chosen so that $r<0$ on $M$ and $r>0$ outside of $\bar{M}$. In a local coordinate chart $U$ of a complex manifold $\bar{M}$, any $C^{\infty}$ vector field can be written as $L=\sum_{j=1}^{2 n} a_{j} \frac{\partial}{\partial x_{j}}$ where $a_{j} \in C^{\infty}(U)$ and $x_{j}, j=1,2, \ldots, 2 n$, are local (real-valued) coordinate functions. Therefore we can define a $C^{\infty}$-metric for a $C^{\infty}$ vector field $L$ by

$$
\|L\|_{C^{\infty}(U)}=\max _{j}\left\{\left\|a_{j}\right\|_{C^{\infty}(U)}\right\}
$$

where $\left\|a_{j}\right\|_{C^{\infty}(U)}$ is a $C^{\infty}$-metric for $C^{\infty}$ functions.

Definition 2.1. Let $I \subset \mathbb{R}^{l}$ be a domain containing 0 . Then the family of complex manifolds $\left\{M_{\tau}\right\}_{\tau \in I}, M_{\tau} \subset M^{\prime}$, with smooth defining functions $r_{\tau}$ for $\tau \in I$, is said to be a continuous family of diffeomorphic complex manifolds with diffeomorphisms $d_{\tau}: M_{\tau} \rightarrow M_{0}$, if

(1) $d_{0}: M_{0} \rightarrow M_{0}$ is the identity,

(2) the complex structures on $M_{\tau}$ are $C^{\infty}$ close to the complex structure on $M_{0}$ as $\tau \rightarrow 0$,

(3) $r_{\tau}$ and all of its derivatives are continuous functions with respect to $\tau$,

(4) the diffeomorphisms $d_{\tau}$ are continuous functions of $\tau$.

Let $\|\cdot\|_{m, t}$ be the $m$-th order weighted Sobolev norm with weight $e^{-t \varphi}$ and let $H^{0,1}\left(M_{\tau}\right)$ be the $\bar{\partial}$-cohomology group on $M_{\tau}$. Here $\varphi$ is a smooth function on $\bar{M}_{\tau}$ for all $\tau \in I$.

Theorem 2.2 ([5, Theorem 3.4]). Let $\left\{\bar{M}_{\tau}\right\}_{\tau \in I}$ be a continuous family of smoothly bounded diffeomorphic compact pseudoconvex complex manifolds in $M^{\prime}$. Suppose there is a $C^{\infty}$ plurisubharmonic function $\varphi$ defined on a neighborhood of $\cup_{\tau} \bar{M}_{\tau}$ which is strongly plurisubharmonic on a neighborhood of $b M_{\tau}$ for all $\tau \in I$. Then there exists a constant $C_{m, t}$ which does not depend on $\tau$, and there is a neighborhood $I^{\prime}$ of $\tau=0$, such that

$$
\left\|f^{\tau}\right\|_{m, t} \leq C_{m, t}\left\|\square_{\tau} f^{\tau}\right\|_{m, t}
$$

for all $f^{\tau} \in D_{\square_{\tau}} \cap C^{\infty}\left(\bar{M}_{\tau}\right)$ with $f^{\tau} \perp H^{0,1}\left(M_{\tau}\right), \tau \in I^{\prime}$. Here $\square_{\tau}$ denotes the usual complex Laplacian on $M_{\tau}$ and $D_{\square_{\tau}}$ denotes the domain of $\square_{\tau}$.

\section{A proof of the approximation theorems.}

In [6], the second author constructed a continuous family of diffeomorphic strongly pseudoconvex compact complex manifolds which shrinks nicely to a compact pseudoconvex complex manifold of finite type. Such a well-behaved continuous family and a stability theorem for the $\bar{\partial}$ - Neumann problem are ingredients to prove our approximation theorem on a compact pseudoconvex complex manifold of finite type. Also, we make use of the following density lemma. 
Lemma 3.1 ([3, Proposition 3.1.4]). Let $\bar{M}$ be a smoothly bounded compact pseudoconvex complex manifold. Suppose there is a $C^{\infty}$ plurisubharmonic function $\varphi$ on $\bar{M}$ which is strongly plurisubharmonic on a neighborhood of bM. Let $f$ be a function in $H(M) \cap H_{m}(M)$, where $m$ is a nonnegative integer. Then for any $\epsilon>0$, there exists $g \in A^{\infty}(M)$ with $\|g-f\|_{m, M}<\epsilon$.

Proof of Theorem 1.1. By using the bumping theorem [6, Theorem 2.3], Cho constructed a smoothly bounded pseudoconvex complex manifold $\Omega$ in $M^{\prime}$ so that $\bar{M}$ can be holomorphically embedded into $\Omega$. Also in [6, Lemma 4.2], he proved that there exists $\epsilon>0$ such that for all sufficiently small $\delta>0$, there is a smooth plurisubharmonic function $\varphi \in C^{\infty}(\bar{M})$ such that $|\varphi| \leq 1$, and for all smooth vector field $L$,

$$
\begin{gathered}
\partial \bar{\partial} \varphi(L, \bar{L}) \gtrsim \delta^{-2 \epsilon}|L|^{2}, \\
\partial \bar{\partial} \varphi(L, \bar{L}) \gtrsim|L \varphi|^{2} \text { for } z \in S(\delta),
\end{gathered}
$$

where $S(\delta)=\left\{z \in M^{\prime}:-\delta<r(z)<\delta\right\}$. Thus $\varphi$ is strongly plurisubharmonic on $S\left(\delta_{0}\right)$ for some $\delta_{0}>0$. Define $r_{\delta}(z)=r(z)+\delta(\varphi-2)$ and set $\Omega_{\delta}=\{z \in$ $\left.M^{\prime}: r_{\delta}(z)<0\right\}$. Since $|\varphi| \leq 1$, there exists $\delta_{0}>0$ such that $\Omega_{\delta} \subset \bar{\Omega}$ and $\varphi$ is defined near $b \Omega_{\delta}$ for all $\delta \leq \delta_{0}$. Using (3.1), we can prove that each $\Omega_{\delta}$ is a strongly pseudoconvex complex manifold for all $0<\delta \leq \delta_{0}$ provided $\delta_{0}$ is sufficiently small (See [6, Proof of Theorem 1]). Thus $\left\{\bar{\Omega}_{\delta}\right\}_{0<\delta \leq \delta_{0}}$ is a continuous family of diffeomorphic strongly pseudoconvex compact complex manifolds such that $\bar{\Omega}_{0}=\bar{M} \Subset \Omega_{\delta}$ for all $0<\delta \leq \delta_{0}$.

By Lemma 3.1, $A^{\infty}(M)$ is dense in $H(M) \cap H_{m}(M)$. Thus it is enough to prove our theorem in the case of functions $f$ in $A^{\infty}(M)$. Let $f \in A^{\infty}(M)$ and define $f_{\delta}=P_{t}^{\delta}\left(d_{\delta}^{*} f\right)$, where $d_{\delta}: \bar{\Omega}_{\delta} \rightarrow \bar{\Omega}_{0}$ are diffeomorphisms and $P_{t}^{\delta}: L^{2}\left(\Omega_{\delta}, t \varphi\right) \rightarrow$ $L^{2}\left(\Omega_{\delta}, t \varphi\right) \cap H\left(\Omega_{\delta}\right)$ are Bergman projections with respect to the weight $e^{-t \varphi}$. Then $f_{\delta}$ is holomorphic on $\Omega_{\delta}$ and

$$
f_{\delta}=d_{\delta}^{*} f-\bar{\partial}_{\delta}^{*} N_{t}^{\delta} \bar{\partial}_{\delta} d_{\delta}^{*} f
$$

where $N_{t}^{\delta}$ is the Neumann operator on $\Omega_{\delta}$ with respect to the weight $e^{-t \varphi}$. By Theorem 2.2, for any nonnegative integer $m \geq 0$,

$$
\begin{aligned}
\left\|\bar{\partial}_{\delta}^{*} N_{t}^{\delta} \bar{\partial}_{\delta} d_{\delta}^{*} f\right\|_{t, m} & \lesssim\left\|N_{t}^{\delta} \bar{\partial}_{\delta} d_{\delta}^{*} f\right\|_{t, m+1} \\
& \lesssim\left\|\bar{\partial}_{\delta} d_{\delta}^{*} f\right\|_{t, m+1}
\end{aligned}
$$

uniformly in $\delta$ near 0 . Since the complex structures on $\bar{\Omega}_{\delta}$ converge to the complex structure on $\bar{\Omega}_{0}=\bar{M}$ in $C^{\infty}$-topology, we can get $\bar{\partial}_{\delta} d_{\delta}^{*} f \rightarrow \bar{\partial} f=0$, also in $C^{\infty}$ topology as $\delta \rightarrow 0$. So $\left\|\bar{\partial}_{\delta}^{*} N_{t}^{\delta} \bar{\partial}_{\delta} d_{\delta}^{*} f\right\|_{t, m}$ converges to zero as $\delta \rightarrow 0$. Since the diffeomorphisms $d_{\delta}$ are continuous functions of $\delta, d_{\delta}^{*} f \rightarrow f$ in $C^{\infty}$-topology on $\bar{M}$. Notice that the weighted Sobolev space is the usual Sobolev space with a different, but comparable norm. Thus

$$
\lim _{\delta \rightarrow 0}\left\|f-f_{\delta}\right\|_{m, M}=0 .
$$

This proves the theorem 1.1.

By extremal properties of the Bergman kernel function, the holomorphic approximation theorem implies the interior stability of the Bergman kernel function (See [10]). Thus we obtain the following theorem as a corollary of Theorem 1.1: 
Theorem 3.2. Let $D_{0}$ be a smoothly bounded pseudoconvex domain in $\mathbb{C}^{n}$ of finite type in the sense of D'Angelo. Let $C$ be a compact subset of $D_{0}$. Given $\epsilon>0$, there exists a domain $D$ containing $\bar{D}_{0}$ for which

$$
\sup _{z, w \in C \cap D}\left|K_{D}(z, w)-K_{D_{0}}(z, w)\right|<\epsilon .
$$

Proof of Theorem 1.2. In [6, Theorem 2.3], the second author proved that there exist a neighborhood $V$ of $z_{0}$ and a smooth 1-parameter family of compact pseudoconvex complex manifolds $\bar{M}_{\delta}, 0 \leq \delta<\delta_{0}$, each defined by $\bar{M}_{\delta}=\left\{z \in M^{\prime}: r_{\delta}(z) \leq 0\right\}$ and satisfying the following properties:

(1) $\bar{M}_{0}=\bar{M}$.

(2) $\bar{M}_{\delta_{1}} \subset \bar{M}_{\delta_{2}}$ if $\delta_{1}<\delta_{2}$.

(3) $\bar{M}_{\delta}-V=\bar{M}_{0}-V$.

(4) There exist a family of diffeomorphisms $d_{\delta}: \bar{M}_{\delta} \rightarrow \bar{M}_{0}$ such that $d_{0}=I d$ and $d_{\delta}=I d$ on $\bar{M}_{\delta}-V$.

(5) $r_{\delta}$ and all of its derivatives are continuous functions with respect to $\delta$.

For any vector field $L$ near $b M_{\delta}$, it follows that

$$
\partial \bar{\partial} d_{\delta}^{*} \varphi(L, \bar{L})=\partial \bar{\partial} \varphi\left(\left(d_{\delta}\right)_{*} L,\left(d_{\delta}\right)_{*} \bar{L}\right) \geq c\left|\left(d_{\delta}\right)_{*} L\right|^{2}>\frac{c}{2}|L|^{2}
$$

provided $\delta$ is sufficiently small. Thus we may assume that $\varphi$ is strongly plurisubharmonic in a neighborhood of $b M_{\delta}$ for all $0 \leq \delta<\delta_{0}$.

First we assume $f \in A^{\infty}(M)$. Define $f_{\delta}=P_{t}^{\delta}\left(d_{\delta}^{*} f\right)$. Then as in the proof of Theorem 1.1, we can prove that $f_{\delta} \in H\left(M_{\delta}\right) \cap H_{m}\left(M_{\delta}\right)$ and that for any $\epsilon>0$, there exists $\delta^{\prime}<\delta_{0}$ such that

$$
\left\|f_{\delta}-f\right\|_{m, M}<\frac{\epsilon}{2} \text { for all } \delta<\delta^{\prime}
$$

Since $A^{\infty}\left(M_{\delta}\right)$ is dense in $H\left(M_{\delta}\right) \cap H_{m}\left(M_{\delta}\right)$, there exists $f_{\delta}^{\prime} \in A^{\infty}\left(M_{\delta}\right)$ such that $\left\|f_{\delta}-f_{\delta}^{\prime}\right\|_{m, M_{\sigma}}<\frac{\epsilon}{2}$ for each $\delta<\delta^{\prime}$. Thus $f_{\delta}^{\prime} \in H(M) \cap C^{\infty}(\bar{M})$ extends holomorphically across $z_{0}$ and

$$
\left\|f-f_{\delta}^{\prime}\right\|_{m, M}<\epsilon \text { for all } \delta<\delta^{\prime}
$$

Since $A^{\infty}(M)$ is dense in $H(M) \cap H_{m}(M)$, we also get the theorem 1.2.

\section{REFERENCES}

1. F. Beatrous and R. M. Range, On holomorphic approximation in weakly pseudoconvex domains, Pacific J. Math. 89 (1980), 249-255.

2. E. Bedford and J. E. Fornaess, A construction of peak functions on weakly pseudoconvex domains, Ann. of Math. 107 (1978), 555-568.

3. D. W. Catlin, Boundary behavior of holomorphic functions on pseudoconvex domains, Dissertation, Princeton University, 1978.

4. D. W. Catlin, A Newlander-Nirenberg theorem for manifolds with boundary, Mich. Math. J. 35 (1988), 233-240.

5. H. R. Cho, S. Cho, and K. H. Shon, Stability of the estimates for $\bar{\partial}$-equation on compact pseudoconvex complex manifolds, Kyushu J. Math. 48 (1994), 19-34. 
6. S. Cho, Extension of complex structures on weakly pseudoconvex compact complex manifolds with boundary, Math. Z. 211 (1992), 105-120.

7. J. P. D'Angelo, Real hypersurfaces, orders of contact, and applications, Ann. Math. 115 (1982), 615-637.

8. K. Diederich and J. E. Fornaess, Pseudoconvex domains: An example with nontrivial Nebenhülle, Math. Ann. 225 (1977), 275-292.

9. J. E. Fornaess and A. Nagel, The Mergelyan property for weakly pseudoconvex domains, Manuscripta Math. 22 (1977), 198-208.

10. R. E. Greene and S. G. Krantz, Stability of the Bergman kernel and curvature properties of bounded domains, Recent Developments in Several Complex Variables (J. Fornaess, Ed.), Annals of Math. Studies No. 100, Princeton Univ. Press, Princeton, N. J., 1981.

11. G. M. Henkin, Integral representations of functions holomorphic in strictly pseudoconvex domains and some applications, Math. Sb. 78 (1969), 611-632; Engl. Transl.: Math. USSR. Sb. 7 (1969), 597-616.

12. N. Kerzman, Hölder and $L^{p}$ estimates for solutions of $\bar{\partial} u=f$ in strongly pseudoconvex domains, Comm. Pure Appl. Math. 24 (1971), 301-379.

13. I. Lieb, Ein Approximationssatz auf streng pseudokonvexen Gebieten, Math. Ann. 184 (1969), 56-60.

14. S. N. Mergelyan, Uniform approximation to functions of a complex variable, Uspehi Mat. Nauk 7 (1952), 31-122.

15. R. M. Range, Approximation by holomorphic functions on pseudoconvex domains with isolated degeneracies, Manuscripta Math. 20 (1977), 309-313.

(†) Dept. of Mathematics, Edu., Andong National University, Andong 760-749, KOREA

(†) Dept. of Mathematics, Sogang University, Seoul 121-742, Korea

E-mail address: shcho@ccs.sogang.ac.kr 